\title{
Research Progress of Cancer Related Depression in TCM
}

\author{
Yunqi He \\ School of Traditional Chinese Medicine, Jinan University, Guangzhou, China
}

Keywords: Cancer; Depression; Traditional Chinese Medicine; Therapy.

\begin{abstract}
In recent years, the high incidence of cancer-related depression has been associated with many factors. At the same time, the direct cause of death for many cancer patients is not cancer itself, but depression. Therefore, it is necessary to study the harm and treatment of cancer-related depression. This paper reviews the current research status of cancer-related depression at home and abroad, as well as the research progress of Chinese medicine for the treatment of cancer-related depression, and provides some reference for those engaged in related research.
\end{abstract}

\section{Introduction}

According to the statistics of cancer incidence and mortality in 2015, the mortality rate of cancer patients in Chinese cities is about $57 \%$, and even as high as $70 \%$ in countryside[1]. Cancer deaths have become a major public health issue in a country with a large population. Among them, the occurrence, development and prognosis of malignant tumors are closely related to the psychological factors of patients. The phenomenon of depression related to cancer patients has gradually aroused social concern. TCM traditional Chinese medicine has rich experience in treating the manifestations of depression [2-4]. The intervention of TCM can improve the depressive symptoms of cancer patients to a certain extent.

\section{Research Progress of Western Medicine on Cancer-related Depression}

\subsection{Incidence rate}

According to the survey, the incidence of depression among cancer patients reported abroad is about $18 \%-50 \%$ [5]. Depression is a kind of disease mainly caused by low mood due to various reasons, which will lead to a continuous decline in the physical and mental health of the human body. Depression patients are prone to physical reactions such as tiredness, physical pain, palpitation, shortness of breath, dyspnea, anorexia or loss, difficulty sleeping, etc. Their aimless uncontrolled behaviors, such as psychologically, depression, pessimism, anxiety, self-accusation, even hallucinations, delusions and other psychotic symptoms will increase [6].

A total of 131 cancer patients were invited to complete the Hospital Anxiety and Depression Scale survey[7]. For both cancer patients, the strongest independent factor influencing the life was anxiety and depression. Another recent study evaluated the depression of 102 cancer patients during their recovery period. As a result, the anxiety, depression, and psychological distress scores of the cancer patients were generally higher. Against the background of the increasing medical technology, the incidence of depression in cancer patients remains high[8]. Some patients even give up treatment in the extreme way of suicide, which also makes psychological depression a major inducer of cancer patient death [9].

\subsection{Influencing factors}

The high incidence of cancer-related depression is affected by a number of factors. From the perspective of the affected population, the incidence of cancer-related depression in the elderly is higher than in adolescents, and they usually have fewer or more difficult to take active and effective responses[10]. However, The women with cancer who have weaker stress resistance and more sensitive minds are more likely to develop depression than men who are generally adapted to social 
stress[11]. From the medical point of view, if surgical intervention, radiotherapy and chemotherapy methods inevitably cause incomplete body or even postoperative pain, patients will increase anxiety. Alonso J[12] found that chronic cancer pain is the main cause of depression, and persistent or aggravating pain may be a signal of exacerbation in patients with advanced cancer.

Increased side effects and tolerance of anticancer drugs can also discourage patients from fighting cancer. Griffiths Roland R[13] assessed the depression of cancer patients before chemotherapy for the first time, and recorded the patient's adverse drug reaction questionnaire. The results showed that patients with severe depression had higher incidences of nausea, vomiting, and fatigue. It is suggested that depression can aggravate the occurrence and severity of adverse reactions to anticancer drugs.

\subsection{Harm and treatment}

Once a cancer patient has depression, it has many adverse effects. On the one hand, the negative emotions of depression can promote cancer progression and worsen cancer prognosis through neuroendocrine disorders and decreased immune function of the body[14-15]. On the other hand, the patient's neuro-endocrine system is affected, which reduces the body's stress ability and the patient's mental state appears depressed[16-17]. Wang Xin Shelley[18]believes that if cancer patients who have not undergone surgery or drug intervention develop depression, they will promote the spread of cancer cells and increase the risk of death. Even after chemoradiotherapy, it is more difficult for cancer patients to overcome a series of adverse reactions after surgery. Peter[20]and Sam H[21]also found that cancer patients are closely related to depression. Along with the combined pain of adverse reactions and drug side effects, patients' recovery is also challenged.

The mood of caregivers of cancer patients will also affect the mental health of cancer patients[22]. If the caregiver lacks good psychological quality, anxiety and depression occur in the care of cancer patients, which will indirectly induce or aggravate the depression of cancer patients. Shuyu Wei[23] believes that cancer patients have their unique psychological characteristics, and comprehensive nursing interventions can effectively reduce the depression symptoms of patients and improve their quality of life. Chunyan Liao[24], Yi Wu[25] and others proposed to use mindfulness decompression therapy to improve anxiety and depression in cancer patients. However, currently patients still rely on antidepressants. Although there are various nursing measures for cancer patients, they are still slightly lacking in clinical application and efficacy[26-27]. The drug is oriented to prolong patients' lives and cure cancer, but the quality of life of patients has not been significantly improved. Therefore, finding effective antidepressant measures is an important task to reduce the incidence of cancer-related depression.

\section{Advances in TCM research on cancer-related depression}

\subsection{Concept}

In the classics of TCM, "Huang Di Nei Jing" published more than 2000 years ago, there are descriptions of the pathogenesis, appearances and treatment principles that closely conected to the tumors. According to the summaries of theories of TCM, cancer is caused by imbalances between endogenous physical conditions of the body and exogenous pathogenic factors [28-29]. In other words, factors can induce cancer only when the body's own defense system fails. Those pathogenic factors, in Chinese medicine terms, include accumulated toxins, heat and blood stasis, and they attack when a person is in a weak physical condition, without the strength to resist. Furthermore, malfunction of the body-mind communication network may also trigger the development of cancer. So, TCM doctors view cancer is the manifestation of a breakdown in the body's ability to handle pathogenic factors, not a local disease of cells or organs. And emotional abnormal fluctuations can directly or indirectly affect the body's internal organs [30].

TCM treats depression syndrome as a symptom, which has broad and narrow meanings. In a narrow sense, it refers to the symptoms of emotional depression [31]. In a broad sense, depression syndrome is a comprehensive disease caused by all internal environmental disorders. Its main 
symptoms are mental, diet, sleep, behavior, language, and sensory disorders, which are similar to the main symptoms of depression in western medicine[32]. Depression is mainly caused by the liver, and is also closely related to the heart, spleen, and kidneys. TCM explains that the concept of liver can store blood and mainly promote circulation. Normal liver function is the main basis for emotional relaxation. In contrast, depression prevents the normal functioning of the liver, and the body suffers from endocrine disorders. When cancer patients suffer from abnormal emotional disturbance at the same time, depression will aggravate their internal environment disorders, and further produce systemic symptoms related to other organs such as insomnia and poor appetite[33].

\subsection{Intervention}

The treatment philosophy and strategy of TCM emphasizes holistic modulation and improvement of the whole body rather than removing the tumor mass or killing the cancerous cells. Accordingly, the focus of treatment is extending the life expectancy and improving the quality of life of the patient; in other words, the focus is on the patient not the tumor mass. Menglin Li[34] evaluated the efficacy and safety of 12 Chinese herbal preparations (CHM) for treating depression symptoms in cancer patients, and the results showed that CHM had fewer side effects than the conventional antidepressant group. Through continuous research on the treatment of cancer-related depression by traditional Chinese medicine, the exact efficacy and advantages of traditional Chinese medicine have gradually emerged[35]. Leiyang Zhang[36] believes that clinically used antidepressants are mostly chemical synthetic drugs that target monoamine neurotransmitters, while the traditional Chinese medicine compound with antidepressant effects has fewer side effects, mainly relieve gas stasis and calm the spirit and phlegm-blood circulation and other drugs. According to the principle of "treatment based on symptom pattern differentiation", doctors personalize antidepressant treatment for patients with cancer-related depression. The purpose of individualized treatment is to improve the quality of life and survival rate of cancer patients and reduce the incidence of depression.

The mechanism of antidepressant action of Chinese medicine has become a research hotspot[37]. There were 86 patients with pain and depression in advanced esophageal cancer[38]. On the basis of anticancer, paroxetine hydrochloride was selected for the antidepressant in the control group. The observation group was given standard TCM combined with standardized care based on the control group. The efficiency was 73. 81\%, which was higher than the control group. Deng Yuanfan Deng[39] used Zung-SDS and HAMD to score 64 patients with cancer depression. The treatment group received "Bupleurum plus Dragon Bone and Oyster Shell Decoction(Chaihu Jia Longgu Muli Decoction)" and the control group took paroxetine hydrochloride tablets. The results suggest that the two control groups are similar, but the experimental group has no gastrointestinal adverse reactions. Jianjun Zheng[40] randomly divided 126 patients with cancer-related depression into a treatment group and a control group. The treatment group was treated with "Chaihu Shugan Powder". The control group received only psychological treatment. As a result, the total effective rate of the experimental group was $98.2 \%$ and the control group was $85.7 \%$. This shows that Chinese medicine has obvious clinical effect in treating cancer-related depression.

Variously, the external treatment method of traditional Chinese medicine also has its unique advantages, such as acupuncture and ear acupuncture. Acupuncture has the advantages of quick onset, safety, and convenient operation. Guilin Liu[41]used acupuncture and magnetic beads to auricular points to treat 20 cancer patients with insomnia due to cancer-related depression, with a cure rate of $85 \%$, which improved the quality of life of cancer patients. Experiments have further confirmed the effect of acupuncture on patients with depression and insomnia, compared with western medicine treatment, fewer adverse reactions occurred[42-43]. For cancer patients, reducing adverse reactions can reduce their physical burden and promote disease recovery. To sum up, oral and external treatment with TCM characteristics have better curative effect on cancer-related depression, which is obviously better than independent psychological therapy[44]. 


\section{Conclusion}

In recent years, improvement of quality of life and prolongation of survival time of cancer patients, whether the cancerous carcinoma disappears or not in the body, have become important factors for evaluating the benefits of any intervention for cancer patients. With the transition from the bio-medical model to the bio-psycho-social medical model, and the rapid development of lifestyle medicine and social psychology, the role of psychological factors in the occurrence, development and prognosis of cancer diseases has gradually attracted attention. In addition to the fear of cancer, patients' physical and mental health are susceptible to factors such as surgery, radiation therapy, chemotherapy and other factors that cause mental disorders. Depression is most common.

Cancer patients often develop chronic, clinically significant symptoms of depression and anxiety. Although the existing antidepressants and related adjuvant treatments have reduced the incidence of cancer depression to some extent, the effect still needs to be improved. The search for new treatments that can effectively improve the depression symptoms of cancer patients with less side effects has become a research bottleneck. TCM doctors make the diagnosis and prepare a treatment scheme based on the assessment of the pattern of symptoms manifest in each individual. The effect of Chinese herbal medicine on cancer depression is obvious. Besides, some of the biological mechanisms of Chinese herbal medicine's antidepressant need to be further explored [36]. Whether its use conflicts with common anticancer western medicine needs more clinical trials to study.

\section{References}

[1] Wanqing C, Rongshou Z, Baade Peter D, et al. Cancer statistics in China, 2015[J]. CA: a cancer journal for clinicians, Vol. 66(2016) No. 2, p. 115-132.

[2] JongXin D, HuiJie L, GuangWei L, et al. Research progress of TCM in treating tumor-related depression[J]. Shandong Journal of Traditional Chinese Medicine, vol. 38(2019) No. 5, p498-501.

[3] Menglin L, Zijie C, Zhenzhu L, et al. Twelve Chinese herbal preparations for the treatment of depression or depressive symptoms in cancer patients:a systematic review and meta-analysis of randomized controlled trials[J]. BMC complementary and alternative medicine, vol. 19(2019) No. 1, p. 28.

[4] XiaoHua W, Fang L, HaiJiao C. Research progress of non-drug therapy for cancer-related depression in traditional Chinese medicine [J]. World Latest Medicine Information, vol. 19(2019)No. 28, p. 121-122.

[5] Niedzwiedz Claire L, Knifton Lee, Robb Kathryn A, et al. Depression and anxiety among people living with and beyond cancer:a growing clinical and research priority[J]. BMC cancer, vol. 19(2019)No. 1, p. 943.

[6] JianYun W, Min L. Application of cancer pain nursing inpatients with advanced tumor chemoradiotherapy[J]. China Modern Medicine, vol. 26(2019)No. 30, p. 240-243.

[7] Qiuping L, Yinghua X, Huiya Z, et al. Factors influencing the health-related quality of life of Chinese advanced cancer patients and their spousal caregivers:a cross-sectional study[J]. BMC Palliative Care, vol. 15(2016)No. 1, p. 72.

[8] YingGao Chen, Bin Guo, CunLiang Hu, et al. Analysis of influencing factors of quality of life of cancer patients in rehabilitation period[J]. Modern Diagnosis and Treatment, vol. 26(2015)No. 3, p. 557-558.

[9] Juan Z, XiaoDong N. Correlation between social support and life satisfaction in hospitalized patients with advanced cancer[J]. Chinese Evidence-Based Nursing, vol. 5(2019)No. 1, p. 58-61. 
[10] ChaoHui Z, HuiFang L, YanXi C. Survey and countermeasures of anxiety and depression of home caregivers in elderly patients with advanced cancer[J]. Chinese General Practice Nursing, vol. 16(2018)No. 16, p. 1932-1933.

[11] Yue Z, XiaoYue Y, Ling J, et al. Relationship between personality and anxiety and depression in female cancer patients[J]. Journal of Nongken Medicine, vol. 40(2018)No. 1, p. 27-31.

[12] Alonso J, Vilagut G, Chatterji S, et al. Including information about co-morbidity in estimates of disease burden: results from the World Health Organization World Mental Health Surveys[J]. Psychol Med, vol. 41(2011)No. 4, p. 873-886.

[13] Griffiths Roland R, Johnson Matthew W, Carducci Michael A, et al. Psilocybin produces substantial and sustained decreases in depression and anxiety in patients with life-threatening cancer:A randomized double-blind trial[J]. Journal of psychopharmacology (Oxford, England), vol. 30(2016)No. 12, p. 1181-1191.

[14] QiWen C, XueYan O, Lian Z, et al. Effects of bad mood on quality of life and immune function after chemotherapy in breast cancer patients[J]. The Practical Journal of Cancer, vol. 34(2019)No. 9, p. 1478-1481.

[15] Vodermaier Andrea, Linden Wolfgang. Social support buffers against anxiety and depressive symptoms in patients with cancer only if support is wanted: a large sample replication. [J]. Supportive care in cancer: official journal of the Multinational Association of Supportive Care in Cancer, 2019.

[16] ZhengWei L. Analysis of the Effect of Comprehensive Psychotherapy on Improving the Mental State of Cancer Patients[J]. Psychological Monthly, vol. 14(2019)No. 12, p. 6-7.

[17] Ross Stephen, Bossis Anthony, Guss Jeffrey, et al. Rapid and sustained symptom reduction following psilocybin treatment for anxiety and depression in patients with life-threatening cancer:a randomized controlled trial[J]. Journal of psychopharmacology (Oxford, England), vol. 30(2016)No. 12, p. $1165-1180$.

[18] Xin Shelley W, Fengmin Z, Fisch Michael J, et al. Prevalence and characteristics of moderate to severe fatigue: a multicenter study in cancer patients and survivors[J]. Cancer, vol. 120(2014)No. 3, p. 425-32.

[19] XianYong S, JinChun W. Relationship between depression symptoms and quality of life and prognostic factors in patients with oral cancer[J]. Journal of International Psychiatry, vol. 45(2018)No. 5, p. 895-897+917.

[20] Peter, Laszlo. The biological background of the comorbidity of depression and cancer[J]. Neuropsychopharmacol Hung, vol. 20(2018)No. 2, p. 59-64.

[21] Sam H. Ahmedzai, Mary Jocylyn Bautista, Kamel Bouzid, et al. Optimizing cancer pain management in resource-limited settings[J]. Supportive Care in Cancer, vol. 27(2019)No. 6, p. 2113-2124.

[22] Miao W, MiLu W. Clinical research progress in malignant tumor depression[J]. Journal of Modern Oncology, vol. 24(2016)No. 8, p. 1315-1317.

[23] ShuLi W. Analysis of psychological characteristics of cancer patients and nursing countermeasures[J]. Electronic Journal of Clinical Medical Literature, vol. 5(2018)No. 98, p. 188.

[24] ChunYan L, LiNa Q, Hui F, et al. Effects of mindfulness decompression therapy on perceived stress and anxiety and depression in patients with colon

[25] cancer[J]. Chinese Journal of Clinical Oncology and Rehabilitation, vol. 23(2016)No. 8, p. 992-996. 
[26] Yi W, YongHui H, JiaXin Zhang, et al. Meta-analysis of the effects of mindfulness decompression therapy on anxiety and depression in cancer patients in China[J]. Chinese General Practice Nursing, vol. 17(2019)No. 22, p. 2705-2709.

[27] YaLing W, Qian L, YuQin W, et al. Research progress on self-management interventions for pain symptoms in cancer patients[J]. Chinese Journal of Pain Medicine, vol. 25(2019)No. 5, p. 387-390.

[28] Maindet Caroline, Burnod Alexis, Minello Christian, et al. Strategies of complementary and integrative therapies in cancer-related pain-attaining exhaustive cancer pain management[J]. Supportive care in cancer:official journal of the Multinational Association of Supportive Care in Cancer, vol. 27(2019)No. 8, p. 3119-3132.

[29] HongLi Q, ZhengQuan F, KeQun C. Clinical experience of TCM in emotional diseases of patients with tumors[J]. Zhejiang Journal of Integrated Traditional Chinese and Western Medicine, vol. 29(2019)No. 12, p. 1033-1035+1046.

[30] JianYian D, WenYing P, ChengZhan W, et al. Etiology mechanism of breast cancer with depression and its treatment progress[J]. Journal of International Psychiatry, vol. 46(2019)No. 5, p. 784-786.

[31] XiaoRu Y, Xun C, PeiTong Z. Research Status of Risk Factors Related to Cancer Depression in Recent Ten Years[J]. Journal of Traditional Chinese Medicine, vol. 59(2018)No. 19, p. 1694-1698.

[32] ZhiHuan Z, HanYu W, Yan M, et al. A Review of Studies on Classical Chinese Medicine Prescriptions for Depression[J]. Journal of Liaoning University of Traditional Chinese Medicine, vol. 21(2019)No. 1, p. 70-72.

[33] LingHong Y, QiuHua Z, XiuFei G. Research progress of breast cancer associated with depression[J]. Journal of Zhejiang Chinese Medical University, vol. 43(2019)No. 4, p. 387-392.

[34] YuXing Y, Pin Kang W. The underlying causes and pathogenesis of depression complicated by cancer[J]. Chinese Archives of Traditional Chinese Medicine, vol. 32(2014)No. 2, p. 271-273.

[35] Menglin L, Zijie Ch, Zhenzhu L, et al. Twelve Chinese herbal preparations for the treatment of depression or depressive symptoms in cancer patients: a systematic review and meta-analysis of randomized controlled trials[J]. vol. 19(2019)No. 1, p. 28.

[36] JiPeng Z, Ping G, XueWei Z, et al. Analysis of curative effect of TCM on tumor associated with depression[J]. Systems Medicine, vol. 3(2018)No. 2, p. 141-143.

[37] LeiYang Z, Jian J, Min H, et al. Progress in pharmacological research of antidepressant Chinese medicine[J]. Chinese Journal of Experimental Traditional Medical Formulae, vol. 23(2017)No. 24, p. 224-234.

[38] RongXian Z, Lei X, Na W. Research progress on antidepressant mechanism of TCM[J]. Journal of Yunnan University of TCM, vol. 42(2019)No. 1, p. 98-102.

[39] SuYa H, YuQiang W, DaPeng L, et al. Observation on the effect of TCM combined with standardized nursing on pain and depression in patients with advanced esophageal cancer[J]. Electronic Journal of Practical Clinical Nursing Science, vol. 4(2019)No. 17, p. 3-4+6.

[40] YuanFan D. Clinical observation of Chaihu Longgu decoction in the treatment of depression after chemotherapy for malignant tumors[J]. Acta Chinese Medicine and Pharmacology, vol. 27(2018)No. 12, p. 34-35.

[41] JianJun Z, XinHua W, GuangMing L. Chaihu Shugan Powder for Treatment of 126 Cases of Depression[J]Shandong Journal of Traditional Chinese Medicine, vol. 21(2017)No. 9, p. 530. 
[42] GuiLin L. Clinical Study on Acupuncture and Auricular Acupoints for Depression and Insomnia in Cancer Patients [J]. Asia-Pacific Traditional Medicine, vol. 12(2016) No. 7, p. 112-113.

[43] Qiu Y. Clinical Evaluation of Acupuncture Treatment of Insomnia with Depression and Anxiety [J]. Journal of China Prescription Drug, vol. 17(2019) No. 6, p. 124-125.

[44] LiuDi Y, YuMei W. Research progress on multiple mechanisms of acupuncture for depression [J]. World Latest Medicine Information, vol. 18(2018) No. 92, p. 53-54+57.

[45] YiJu Pan, Chi Cheng, LingLing Y, et al. Utilization of traditional Chinese medicine in patients treated for depression:A population-based study in Taiwan[J]. Complementary Therapies in Medicine, vol. 21(2013) No. 3, p. 215-223. 